\title{
Observing Ultra-High Energy Cosmic Rays using Camera Image Sensors
}

\section{Wakiko Takano and Kinya Hibino}

Kanagawa University,

Rokkakubashi 3-27-1, Yokohama, Japan

E-mail: r201970105fg@jindai.jp

\begin{abstract}
We propose a new approach for observing UHECR by detecting charged particles in the core region of EAS using a cost-effective and compact detector with a CMOS camera image sensor. In general, the core region of EAS is excluded from the measurement due to the very high particle density at which the signal saturates. However, the results of the EAS simulation predict that the particle density in the core depends on the distance from the axis and the angular distribution depends on the arrival direction of the primary cosmic ray. Therefore, if we have a detector that does not saturate even in high-density areas, the core might be useful for collecting information about EAS effectively. Meanwhile, CMOS Camera image sensors are sensitive to ionizing radiations in addition to optical photons. The length of the track in the digital images is related to depletion thickness and incident angle to the sensor's surface. When depletion thickness is evident, we can reconstruct the incident angles of primary particles roughly. Since these sensors are thin and small, they have the advantage of being able to observe without saturation, even in high-density regions. We demonstrate the simulation result to show the characteristics of UHECRs core and the reaction of sensors for charged particles. We also report the thickness of the depletion layer estimated from our experimental results using a prototype of the CMOS sensors with Raspberry PI .
\end{abstract}

$37^{\text {th }}$ International Cosmic Ray Conference (ICRC 2021)

July 12th - 23rd, 2021

Online - Berlin, Germany 


\section{Introduction}

Cosmic rays with energies above $10^{18} \mathrm{eV}$ are called ultra-high energy cosmic rays (UHECR), which can be detected only through the extensive air showers (EAS) they produce in the atmosphere. An EAS may consist of billions of secondary particles that arrive at ground level over many square kilometers. Very high-energy primaries arrive at the top of the atmosphere at a rate of about one event per square kilometer per century. And the origin of these ultra-high energy cosmic rays remains a puzzle even many decades after their discovery. Increasing the data statistics is very important, but the small flux requires a large observation area and long-term observation time. To increase more data in a shorter time, we need an idea to expand the observation area at a low cost.

On the other hand, over 1 billion smartphones are in widespread use worldwide, and each device is equipped with a CMOS camera image sensor. Also, tablets, laptops, and mobile devices of all kinds have the same sensor. The CMOS camera image sensor is one of the photodiode sensors that can detect optical photons and ionizing radiation, including cosmic rays. Although it is not developed for scientific purposes, it is important to evaluate the sensor's performance and discuss the possibility of using this sensor as an array to cover the entire surface of the globe.

A few cosmic ray experiments have been performed using a detector with the CMOS camera image sensor. One is DECO[1], which is a citizen science project that enables users around the world to detect cosmic rays and other energetic particles with their cell phones and tablets. Another is called CRAYFIS[2], a novel approach to observe cosmic ray particles at the highest energies. It uses the worldwide array of existing smartphones instead of building an expensive dedicated detector. Also, CREDO[3] is from Poland looking for super-heavy dark matter's photon-induced showers which arrive simultaneously on earth. As these existing studies point out, observing UHECR EAS requires a wide range of high-density devices, which is not an easy task, but we only focus on the EAS core. Since only the core is the observation target, a large effective area can extend while reducing required sensors.

\section{Detection Method}

Figure 1 shows the particle density distribution for a simplified vertically incident EAS with the energy of $10^{20} \mathrm{eV}$. The numbers surrounded by colors indicate the number of charged particles that each of the five sensors can detect within a 200-meter radius of the EAS core. The effective area of the sensor is $1 \mathrm{~cm}^{2}$ at this time. The measured density is compared to the known lateral distribution of particle densities to reconstruct the primary energy. This case is for using 5 sensors in one array and targeting $10^{20} \mathrm{eV}$ cosmic rays, but when we are targeting lower energies, we need to place the sensors closer. That means for lower energies, the effective area for one array would be smaller. Simulation is required to determine the number of sensors needed in the array to observe each energy of interest. And from the effective area and the flux of the primary cosmic rays, the required number of arrays can be estimated according to the expected rate of events. For example, to detect more than one EAS of $10^{20} \mathrm{eV}$ per year, we will need 3,200 arrays. 


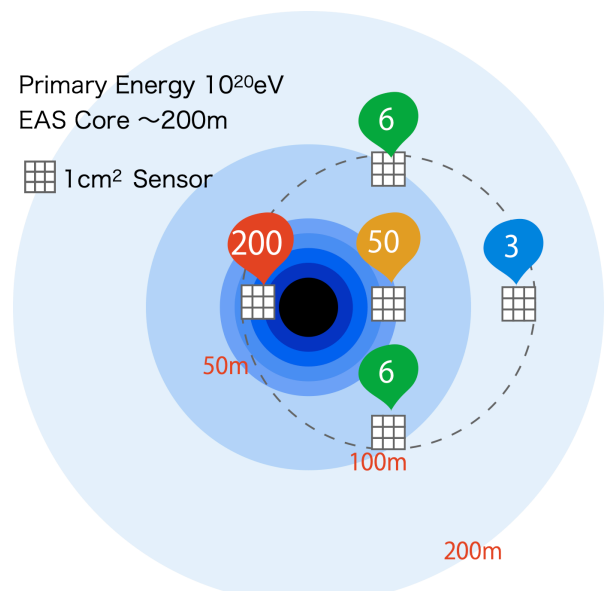

Figure 1: EAS Core. The colored marks indicate the number of particles that can be detected by the $1 \mathrm{~cm}^{2}$ sensor at one time. The red, yellow, green, and blue sensor can observe 200, 50, 6, and 3 particles, respectively, corresponding to a distance of $30 \mathrm{~m}, 50 \mathrm{~m}, 100 \mathrm{~m}$, and $130 \mathrm{~m}$ from the core axis.

\section{Simulation}

\subsection{EAS Core}

We have performed the Corsika simulation to study the UHE air shower core. Figure 2 shows the Lateral distribution of the charged particles in the core. When the energy is $10^{20} \mathrm{eV}$ and the location is within $10 \mathrm{~m}$ of the core, the density is greater than $10^{7}$ particles per $1 \mathrm{~m}^{2}$. This means that more than 1000 particles hit a small sensor of $1 \mathrm{~cm}^{2}$ at a time. The lateral distribution of the particles depends on the energy of the primary cosmic ray. Figure 3 shows the angular distribution of charged particles. The higher the energy of the primary particles, the more the angle distribution concentrates in the incident angle of the primary particles. Figure 4 is the energy distribution of charged particles. The particle with high energy distributes more in the core area.

\subsection{Identifying of Cosmic Ray Signals}

To distinguish the cosmic ray signal from the background, we need to know the characteristics of the cosmic-ray signal. Cosmic rays we observe at ground level are relativistic charged particles. This means cosmic rays penetrate through the sensor depositing energy of minimum ionization loss of leaving trajectories with small dots or straight lines. The brightness of these trajectories would not be too bright because it is proportional to the energy deposited on the silicon sensor. For the dots, it is difficult to distinguish between backgrounds and signals simply by analyzing the shapes or the brightness. Therefore, in this paper, we used only long tracks with straight lines to show the detection of cosmic rays and estimate the depletion thickness of the sensor.

We have performed the Geant 4 simulation to study the CMOS camera image sensor. The reaction of electrons incident on the silicon sensor was investigated. Figure 5 (left) is the energy deposit of electrons. The minimum ionization loss is reached at 0.2 to $1 \mathrm{MeV}$. When the deposited energy is large, the observed trajectories will be large bright spots or curved worms. However, with minimal ionization, the particles are more likely to penetrate the sensor and create small dots or 


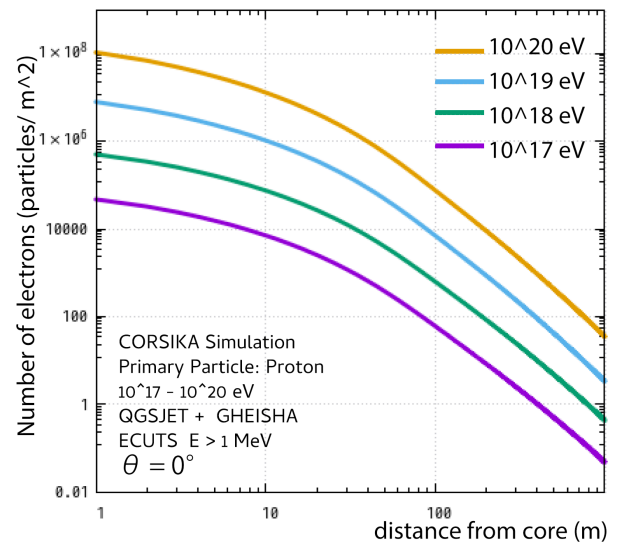

Figure 2: Curves of Lateral distribution of vertical incident EAS for each primary energy.

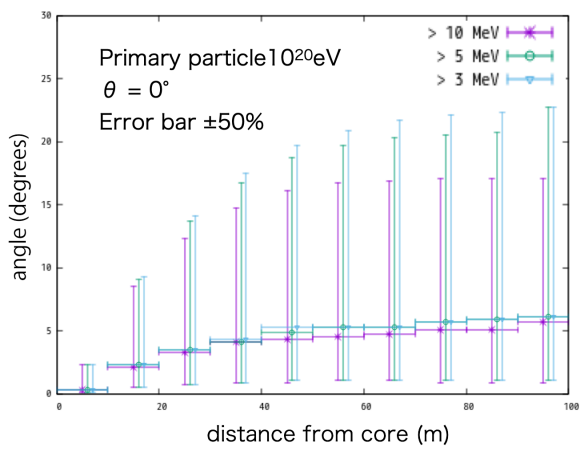

Figure 3: Angular distribution of charged particles in a vertical incident EAS. The mode values of the distribution for each energy threshold were plotted, and the error bar indicated the particles included a number of $\pm 50 \%$.

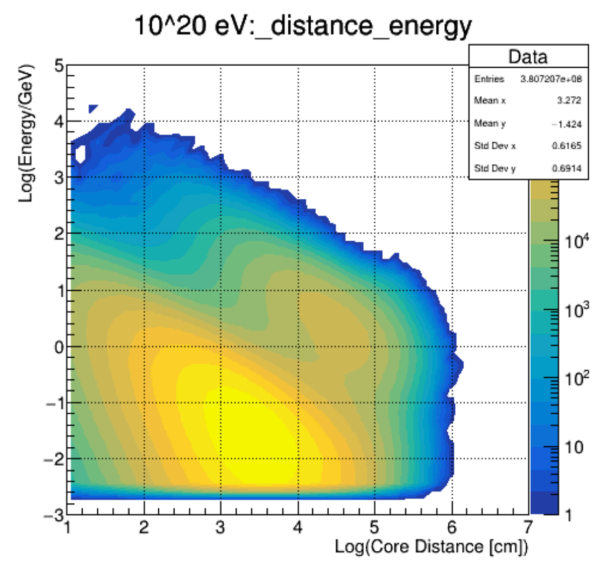

Figure 4: An example of the 2D energy distribution as a function of distance from the core in an EAS with primary energy of $10^{20} \mathrm{eV}$.

short, sharp straight tracks. The length of a track is proportional to the angle of incidence of the particles, as shown in figure 5 (right). The simulation was performed assuming that the thickness of the depletion layer was $20 \mu \mathrm{m}$. However, if the thickness of the depletion layer in the sensor is not correct, the incident angle is not estimated from the length of the track. Therefore, we measured the thickness of the depletion layer of the sensor by using cosmic-ray signals.

\section{Measurement of Cosmic Rays with CMOS Camera Image Sensor and Depletion Thickness}

We measured charged particles using a CMOS camera image sensor. We used a Raspberry Pi 4 with a camera module v2. To prevent visible light from entering the camera, we used black tape 

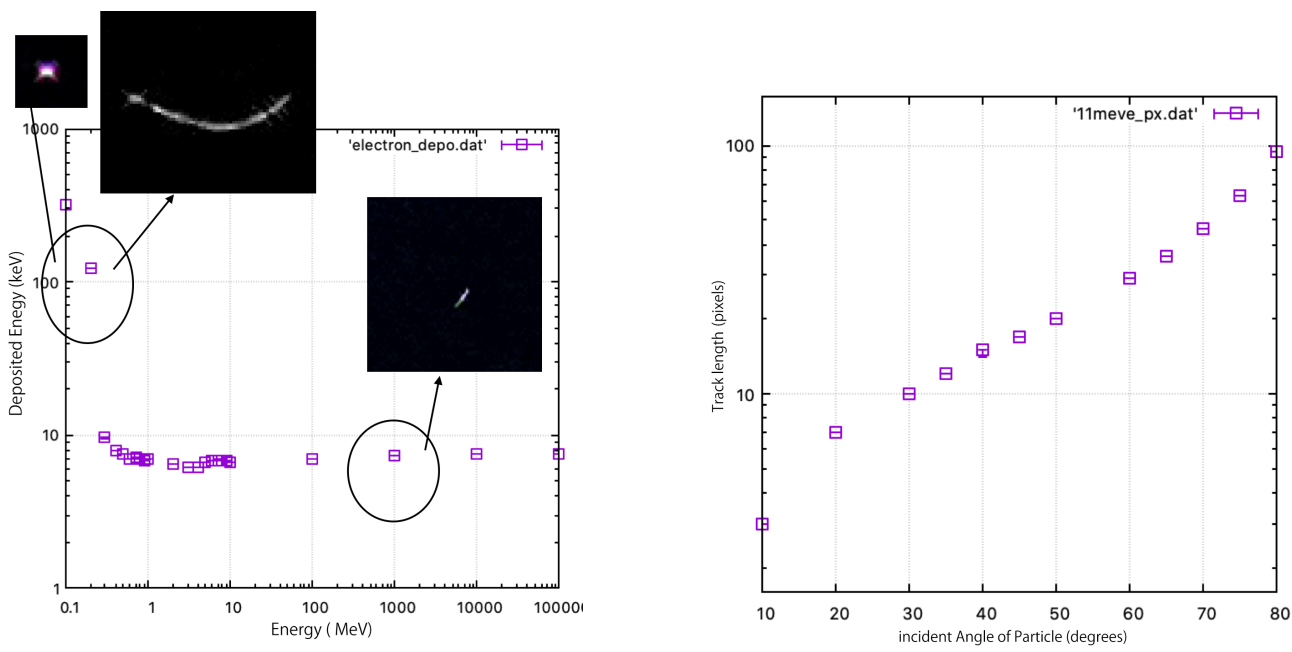

Figure 5: Result by Geant4 Simulation. The left is the energy deposit of electrons. The minimum ionization loss is reached at 0.2 to $1 \mathrm{MeV}$. The right shows that the length of a track is proportional to the angle of incidence of the particles.

to block light. After continuous shooting and noise removal, and then, we analyzed the images.

Figure 6 shows 6 raw images observed by the image sensor. White lines and dots indicate the pixel reactions of charged particles. We classify the event signal by their shapes. Left is a line track, a straight line, the middle is a worm that bends like an earthworm, and a round spot on the right. This time, we used only straight-line tracks to reconstruct the angle of incident particles.

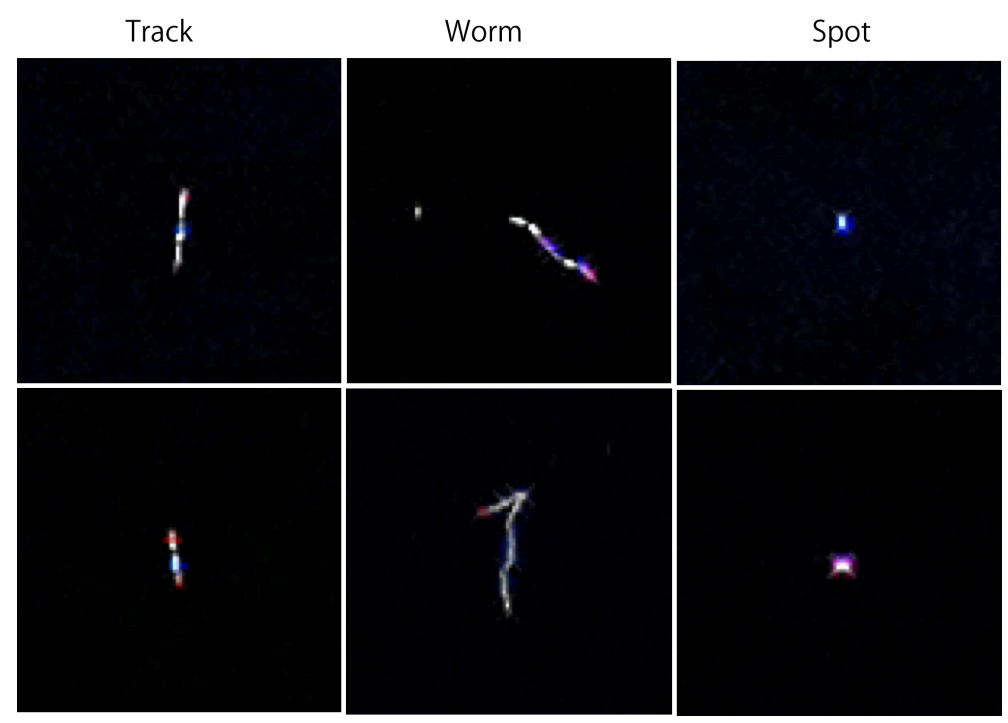

Figure 6: 6 raw images observed by the image sensor and classified by their shapes.

The length distribution of the line track event is fitted by equations (1) and (2) to estimate the thickness of the depletion layer (see the reference [4] in detail). Since these events may include cosmic rays and environmental radiation, we also used an isotropic distribution formula for fitting. 
Equation (1) expressed for the length distribution when it has an isotropic angle distribution, and Equation (2) expressed for the length distribution derived from the zenith angle distribution of cosmic rays. Based on the observed event length $L$, the thickness $H$ of the depletion layer was estimated.

$$
\begin{aligned}
& \frac{d N}{d L}=A \frac{L H^{2}}{\left(L^{2}+H^{2}\right)^{2}} \quad \text { (isotropic) } \\
& \frac{d N}{d L}=B \frac{L H^{4}}{\left(L^{2}+H^{2}\right)^{3}} \quad(\text { cosmic rays })
\end{aligned}
$$
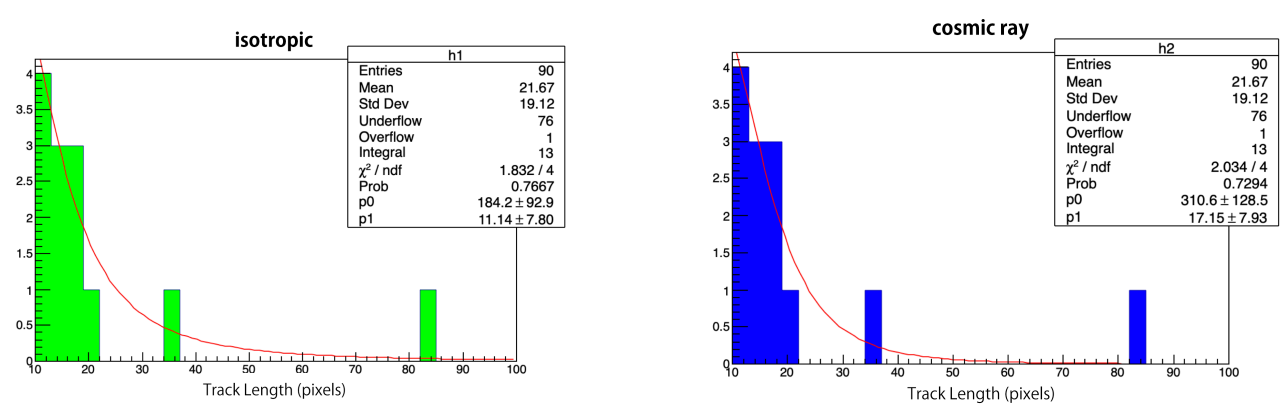

Figure 7: Estimated depletion thickness. The left is the result of fitting using Equation (1).The right is the result of fitting using Equation (2)

We analyzed data that selected single line track events during three days (a total of 90 cases). From the results, we estimated that the thickness of the depletion layer of the sensor Sony IMX2198 used this time is 12 to $19 \mu \mathrm{m}$.

\section{Summary}

We introduced a new idea for observing ultra-high energy cosmic rays, such as using an array consist of a small and thin sensor to form to measure the core of the air shower directly. We also measured the thickness of the depletion layer of the CMOS camera image sensor, and the depletion layer of the sensor used this time was estimated to be $12-19 \mu \mathrm{m}$.

At present, we are accumulating more data and would obtain the depletion layer value more accurately. By reflecting the results in the simulation, we will investigate the method for determining the energy and arrival direction of the primary particles from the observation results.

\section{References}

[1] M. Meehan et al., "The particle detector in your pocket: The Distributed Electronic Cosmic-ray Observatory,", PoS (ICRC2017) 375, 2017.

[2] Albin, E. and Whiteson, D. Feasibility of Correlated Extensive Air Shower Detection with a Distributed Cosmic Ray Network. Arxiv, 2021. 
[3] P. Homola, et al. (CREDO Collab.), Symmetry 2020, 12(11), 1835, 2020.

[4] J. Vandenbroucke et al., "Measurement of camera image sensor depletion thickness with cosmic rays,", Journal of Instrumentation, 11 (04) p. P04019, (2016),. 